development should include complete colonoscopy withdrawals to reflect true clinical practice and focus specifically on identifying challenging polyps that may be overlooked.

\begin{tabular}{ll} 
Abstract OTU-04 Table 1 & \\
\hline Video Dataset & 83 \\
\hline Lesions (n) & 5.4 \\
Mean size (mm) & \\
Morphology (Paris Classification) & 35 \\
Protruded (Ip/lsp/ls) & 48 \\
Flat elevated/Flat (Ila//lb) & \\
Location & 58 \\
Right Colon & 20 \\
Left Colon & 5 \\
Rectum & \\
Pathology & 1 \\
High Grade Tubular & \\
Adenoma & 61 \\
Low Grade Tubular Adenoma & 14 \\
Sessile Serrated Lesion & 7 \\
Hyperplastic Polyp & \\
\hline
\end{tabular}

\section{OTU-05 OUTCOMES OF TRANSANAL ENDOSCOPIC MICROSURGERY (TEM) VERSUS ENDOSCOPIC RESECTION (ER) OF LARGE RECTAL ADENOMAS}

Andrew Emmanuel*, Taimur Shafi, Christo Lapa, Sophie Williams, Shraddha Gulati, Nishmi Gunasingam, Margaret Burt, Savvas Papagrigoriadis, Bu Hayee, Amyn Haji. King's College Hospital NHS Foundtaion Trust, London, UK

\subsection{6/gutjnl-2019-BSGAbstracts.5}

Introduction Resection of large rectal adenomas by TEM or endoscopic resection (ER) varies by institution and region. There is a paucity of data directly comparing these strategies and the procedure of choice remains under intense debatable. We report outcomes of TEM and ER of large rectal adenomas from a large tertiary cohort.

Methods Large $(\geq 20 \mathrm{~mm})$ rectal adenomas resected by TEM or ER (2009-2018) were analysed. From 2009-2011 all rectal adenomas were treated by TEM, after which the primary resection strategy changed to ER. Outcomes were compared between techniques.

Results 258 rectal adenomas were resected by TEM $(n=73)$, $\operatorname{EMR}(n=61)$ and ESD/Hybrid ESD $(n=124)$ with a mean size of $46 \mathrm{~mm}$ for TEM vs $62 \mathrm{~mm}$ for ER (p<0.001). 100\% of patients undergoing TEM required hospital admission vs $22 \%$ for ER $(p<0.001)$, and $100 \%$ required general anaesthesia for TEM vs $13 \% \%$ for ER $(\mathrm{p}<0.001)$. En bloc resection was achieved for $99 \%$ of ESD resections, 52\% of TEMS, $26 \%$ of EMR and $12 \%$ of hybrid ESD $(\mathrm{p}<0.001)$. Complications were similar $(p=0.33)$. Recurrence occurred in $4 \%$ after ESD, $18 \%$ after EMR, 20\% after Hybrid ESD and 42\% after TEM $(\mathrm{p}<0.001)$. No patients undergoing ER required surgery as a result of a complication.

Conclusion Despite significantly larger lesion sizes, ER in our institution provides far superior results for patients than TEM with relatively few patients requiring general anaesthesia or hospital admission and lower recurrence rates especially with ESD. This has led to a policy of ER for all apparently benign rectal tumours regardless of size and, more recently, an ESDfirst approach for all lesions.

\section{OTU-06 UPPER GASTROINTESTINAL BLEEDING IN SCOTLAND: TRENDS IN DEMOGRAPHICS AND OUTCOMES 2000- 2015}

${ }^{1}$ Sardar Chaudhary*, ${ }^{2}$ Daniel Mackay, ${ }^{2}$ Jill P Pell, ${ }^{1}$ Allan J Morris, ${ }^{3}$ Nick Church, ${ }^{4}$ Andrew Fraser, ${ }^{1}$ Adrian I Stanley. ${ }^{1}$ Glasgow Royal Infirmary, Glasgow, UK; ${ }^{2}$ University of Glasgow, Institute of Health and Wellbeing, Glasgow, UK; ${ }^{3}$ Royal Infirmary of Edinburgh, Edinburgh, UK; ${ }^{4}$ Aberdeen Royal Infirmary and QEUH Glasgow, Aberdeen and Glasgow, UK

\subsection{6/gutjnl-2019-BSGAbstracts.6}

Introduction Upper gastrointestinal bleeding (UGIB) remains a common cause of presentation and admission to hospital in the UK, with the incidence in Scotland one of the highest in the world. Over the past 15 years there have been several developments to improve management of patients with UGIB.

Methods Our aim was to investigate the number of patients presenting to Scottish hospitals with UGIB between 20002015 and assess the difference in demographics, aetiology of bleeding and clinical outcomes, including those for weekends and weekday presentations. Data were collected from SMR01 hospital admissions records and GRO death certificates for the period 1st January 2000 to 31st December 2015 and analysed in the national safe haven. All outputs were disclosure-checked for confidentiality purposes. Admission and death rates were computed for each year with trends over the study period estimated using Poisson regression. Standard errors were adjusted to account for any serial dependence.

Results A total of 129,404 patients presented to Scottish hospitals with UGIB between January 2000 and December 2015. Mean age at admission increased over this period from 59.2 years to 61.4 years $(\mathrm{P}=0.049)$. There was no difference in the annual number of patients over the 15 -year period. The incidence of UGIB was highest in the more deprived quintiles, although there was a reduction in incidence in the three most deprived quintiles over the study period (SIMD1; $\mathrm{P}<0.001$ : SIMD2; $\mathrm{P}=0.002$ : SIMD3; $\mathrm{P}=0.001$ ). There was a significant decrease in 30 -day case-fatality from $10.1 \%$ in 2000 to $7.9 \%$ in $2015(\mathrm{p}<0.001)$, which was significant across all the deprivation quintiles $(\mathrm{P}<0.001)$. This reduction was seen for both variceal and non-variceal bleeding (IRR 0.967; P $<0.001$ and IRR 0.980; $\mathrm{P}<0.001$ respectively) with deaths as a proportion of admissions declining fastest in the variceal compared to the non-variceal group $(24.4 \%$ to $14.5 \%$, and $9.8 \%$ to $7.8 \%$ respectively). The mean length of stay also fell from 3.9 days to 2.1 days, an average decline of 0.14 days per year (95\% CI .15612 $.11723 ; \mathrm{P}<0.001)$. There was no difference in 30 -day case-fatality between patients presenting at weekdays or weekends.

Conclusions The number of patients presenting with UGIB to Scottish hospitals annually has remained similar over the 15year study period. However, case-fatality and length of hospital stay has fallen, despite a rise in the mean age of patients. 